\title{
Phase-Dependent Shell Growth and Optical Properties of ZnSe/ZnS Core/Shell Nanorods
}

Jiajia Ning, ${ }^{\dagger}$ Yuan Xiong, ${ }^{\ddagger}$ Stephen V. Kershaw ${ }^{\dagger}$ and Andrey L. Rogach ${ }^{\dagger}, *$

${ }^{\dagger}$ Department of Materials Science and Engineering \& Centre of Functional Photonics, City University of

Hong Kong, Hong Kong SAR, China

\$Department of Chemistry, City University of Hong Kong, Hong Kong SAR, China

Corresponding Author: andrey.rogach@,cityu.edu.hk
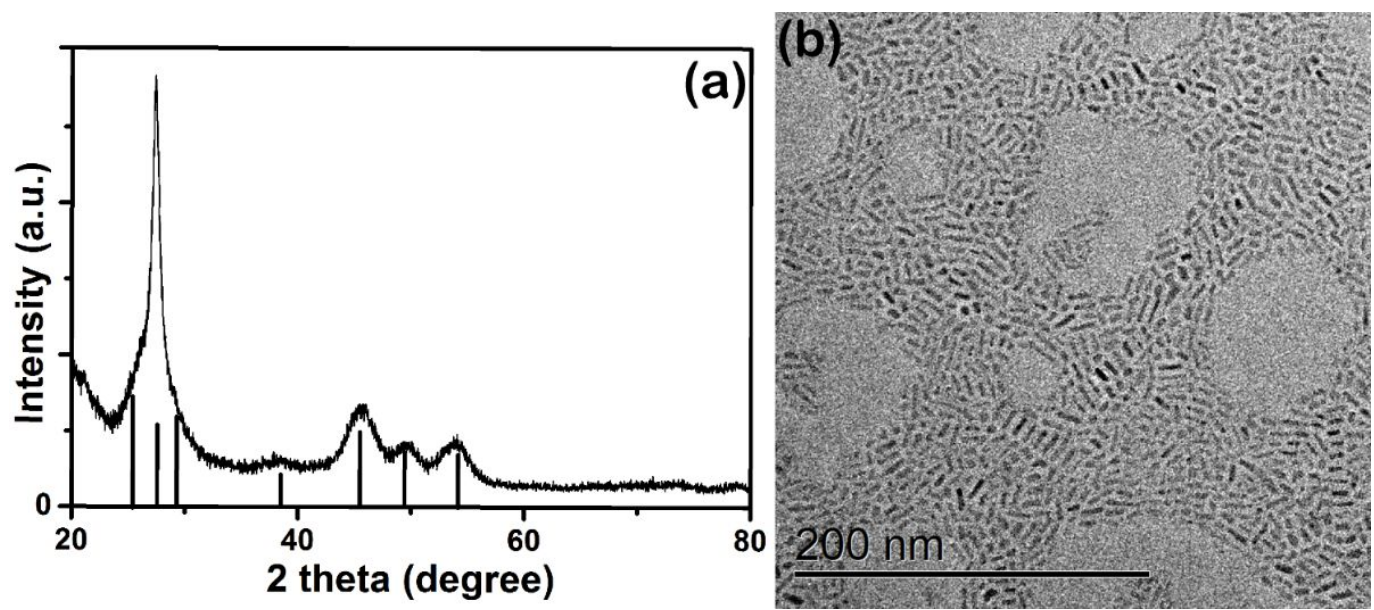

Figure S1. (a) powder XRD pattern and (b) TEM image of WZ-ZnSe NRs; standard diffraction peaks for bulk WZ-ZnSe (PDF\#80-0008) are shown in the bottom. 


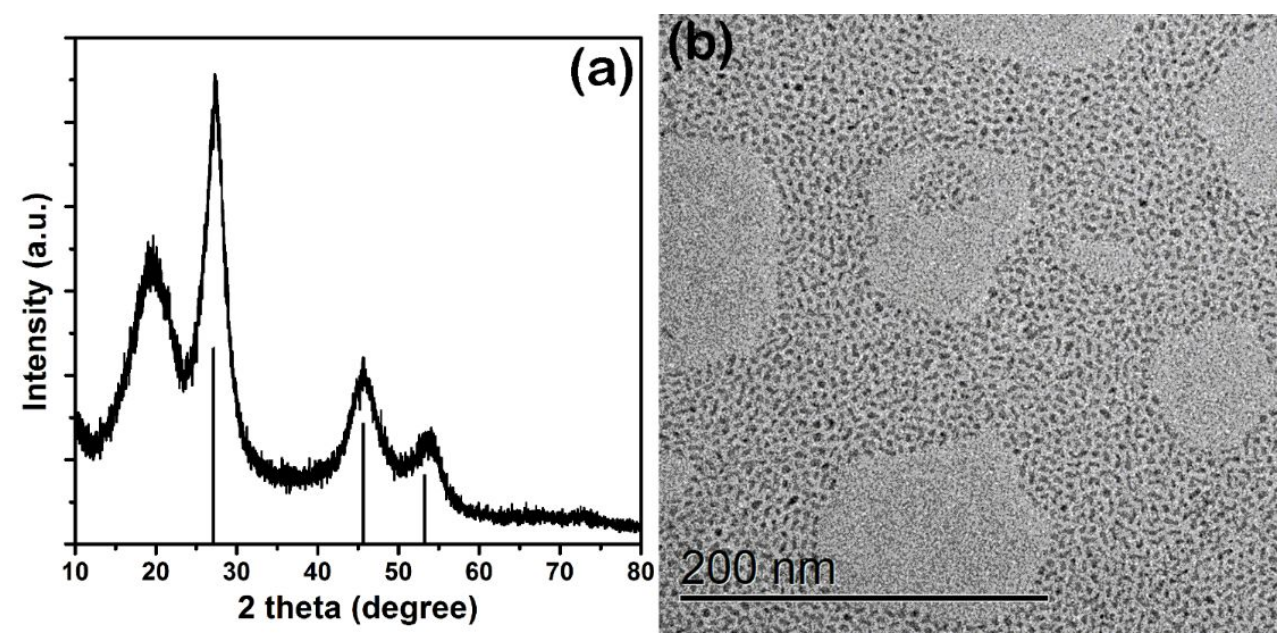

Figure S2. (a) powder XRD pattern and (b) TEM image of ZB-ZnSe NRs; standard diffraction peaks for bulk $\mathrm{Zb}-\mathrm{ZnSe}$ (PDF\#37-1463) are shown in the bottom. 


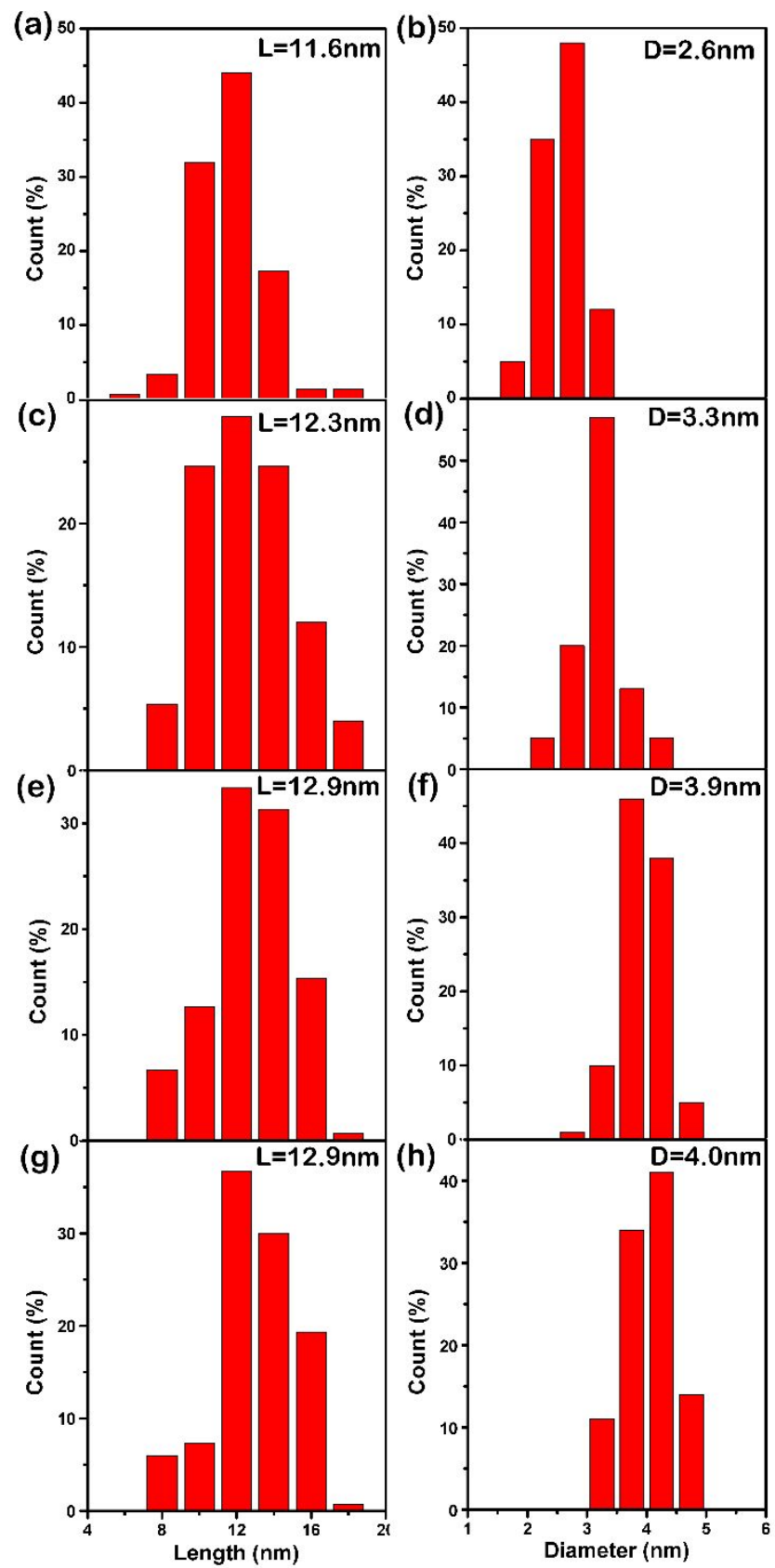

Figure S3. Histograms for lengths (L) and diameters (D) of (a), (b) WZ-ZnSe NRs, and the ZnSe/ZnS core/shell NRs with various thicknesses of ZnS shell: (c), (d) WZ-ZnSe/ZnS core/shell NRs-1.0; (e), (f) WZ-ZnSe/ZnS core/shell NRs-3.0; (g), (h) WZ-ZnSe/ZnS core/shell NRs-5.0. 


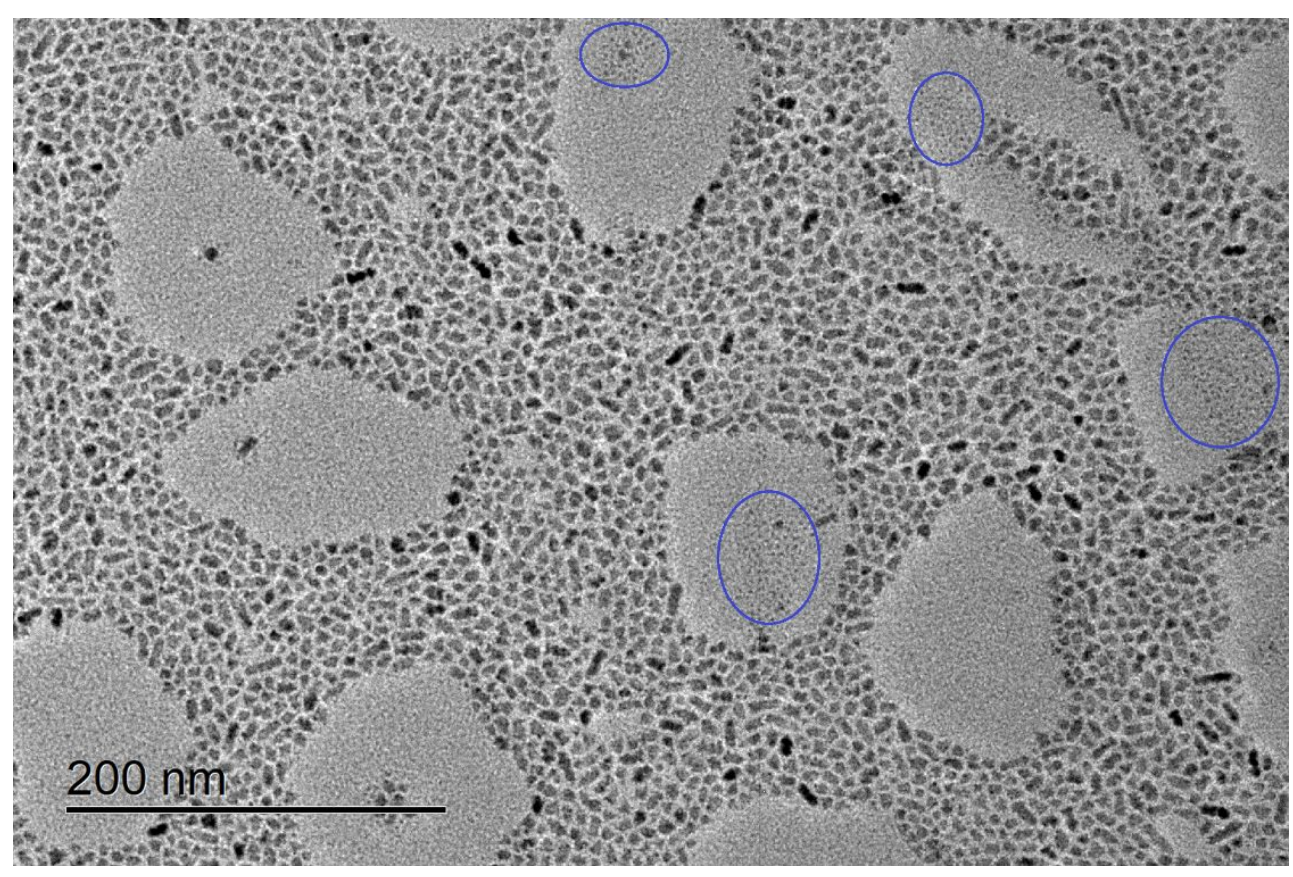

Figure S4. TEM image of WZ-ZnSe/ZnS core/shell NRs-5.0ml; regions on the grid with some dot-shaped ZnS nanoparticles are marked with blue circles. 


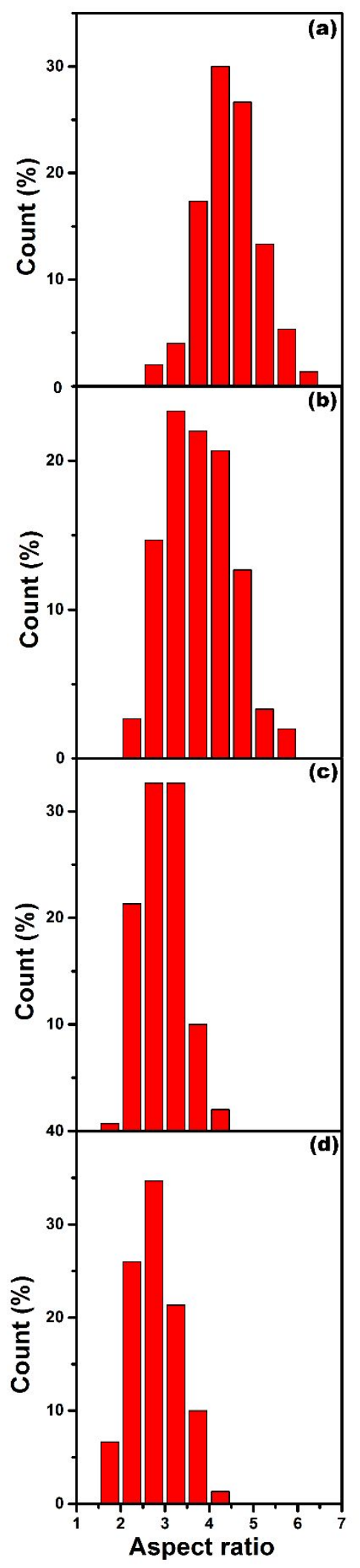

Figure S5. Histograms showing aspect ratio distributions for (a) WZ-ZnSe NRs, (b) WZ-ZnSe/ZnS core/shell NRs-1.0, (c) WZ-ZnSe/ZnS core/shell NRs-3.0, and (d) WZ-ZnSe/ZnS core/shell NRs-5.0. 


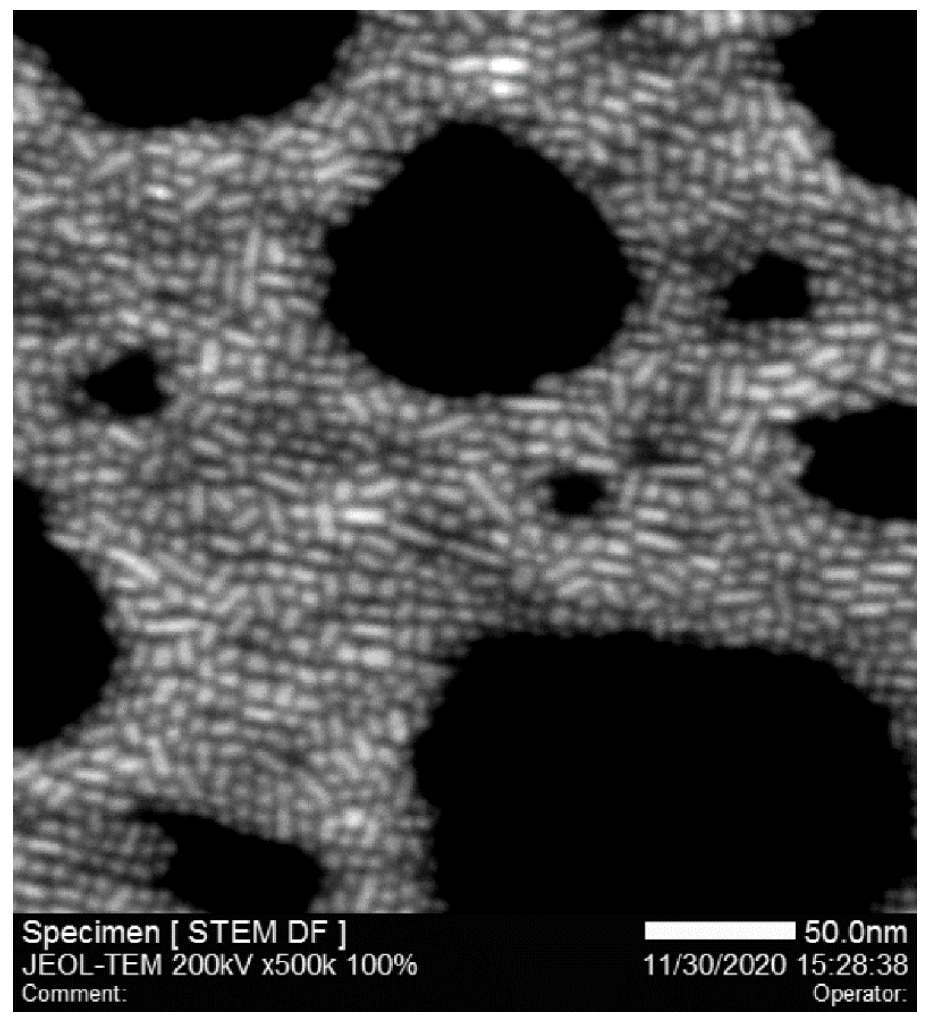

Figure S6. HAADF-STEM image of WZ-ZnSe/ZnS core/shell NRs-3.0. 


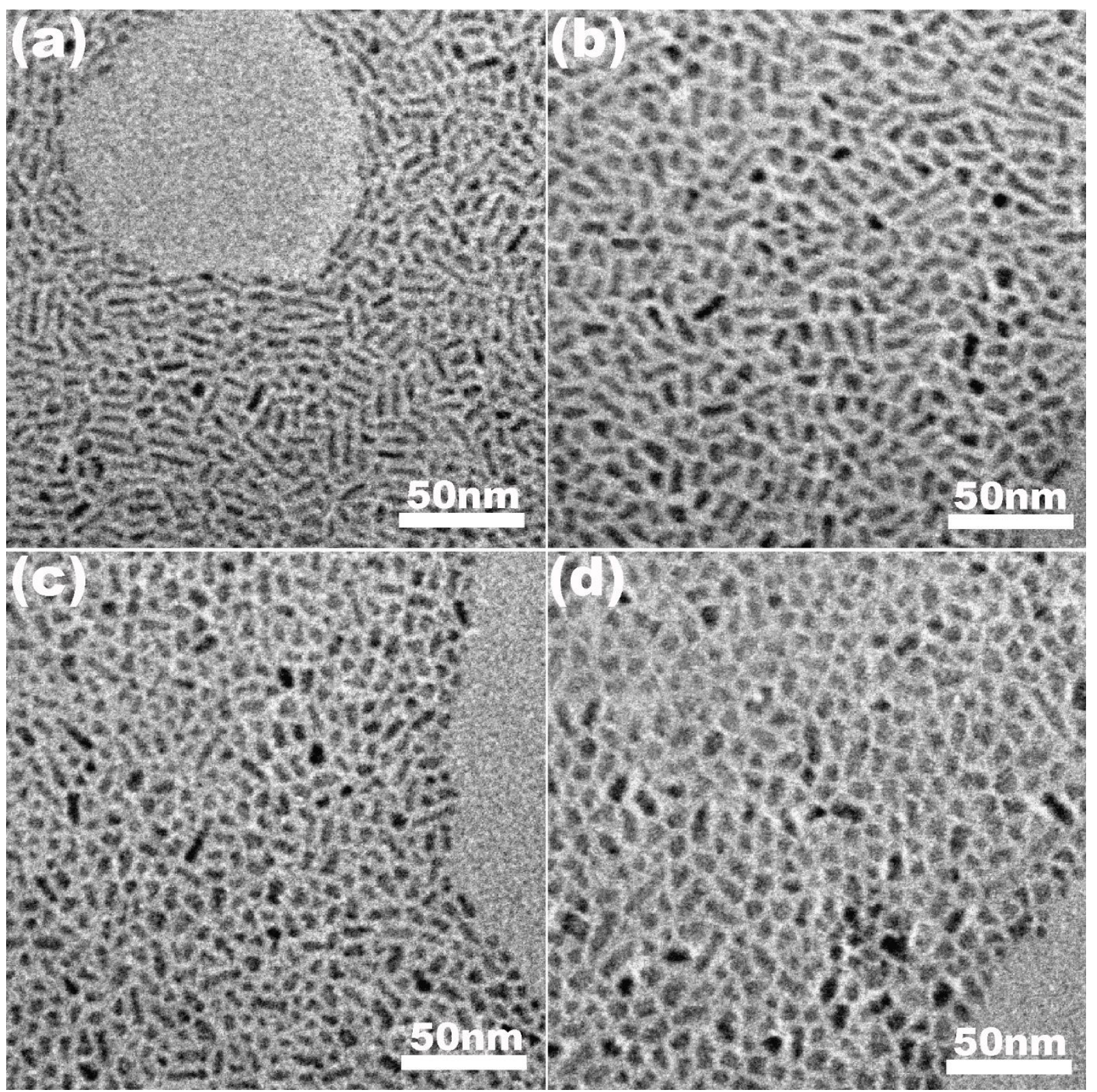

Figure S7. TEM images of (a) L-WZ-ZnSe NRs, (b) L-WZ-ZnSe/ZnS core/shell NRs-1.0, (c) L-WZ-

ZnSe/ZnS core/shell NRs-3.0, and (d) L-WZ-ZnSe/ZnS core/shell NRs-5.0. 

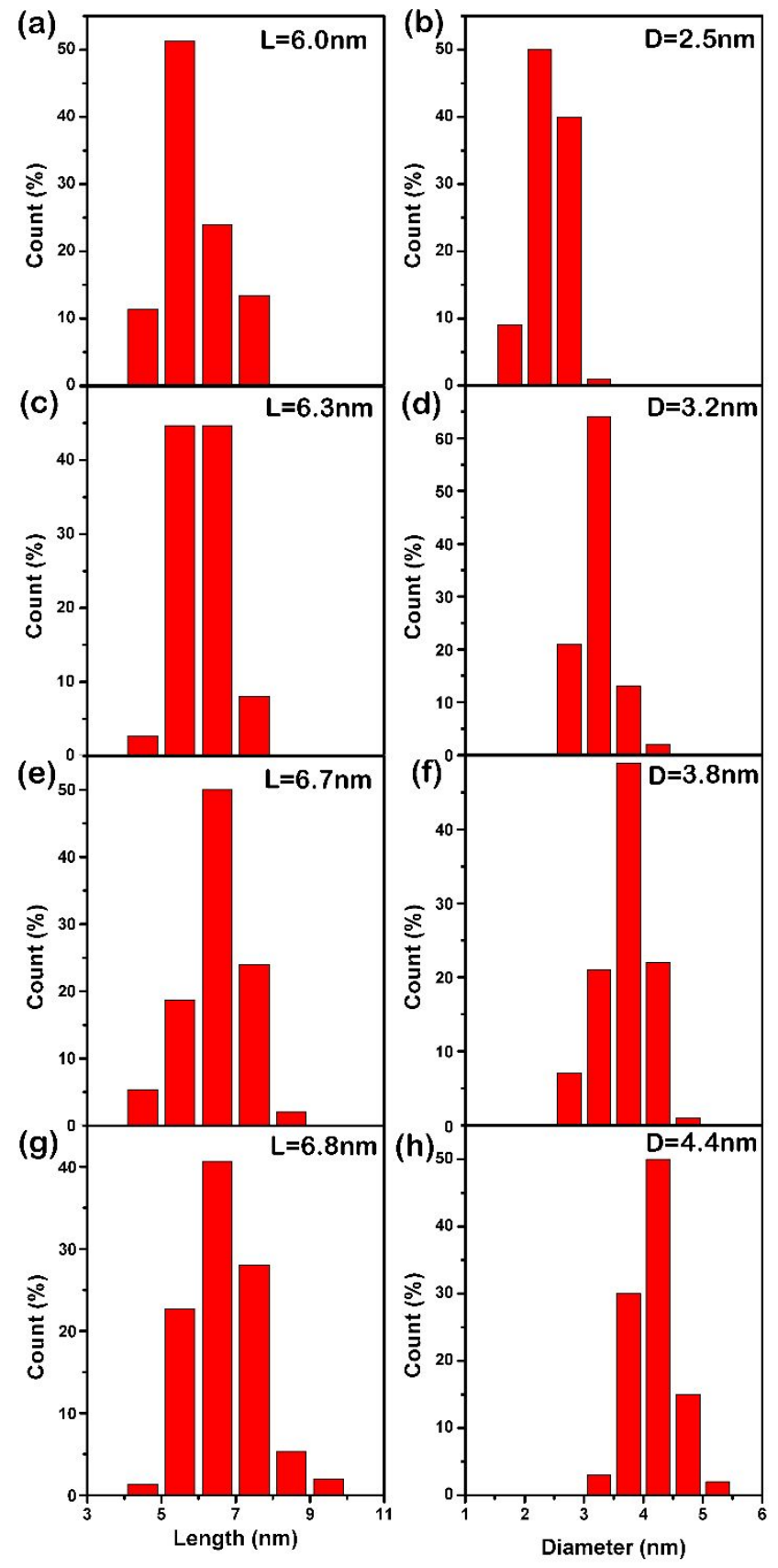

Figure S8. Histograms for lengths (L) and diameters (D) of (a), (b) ZB-ZnSe NRs, and the ZnSe/ZnS core/shell NRs with various thicknesses of ZnS shell: (c), (d) ZB-ZnSe/ZnS core/shell NRs-1.0; (e), (f) ZB-ZnSe/ZnS core/shell NRs-3.0; (g), (h) ZB-ZnSe/ZnS core/shell NRs-5.0. 


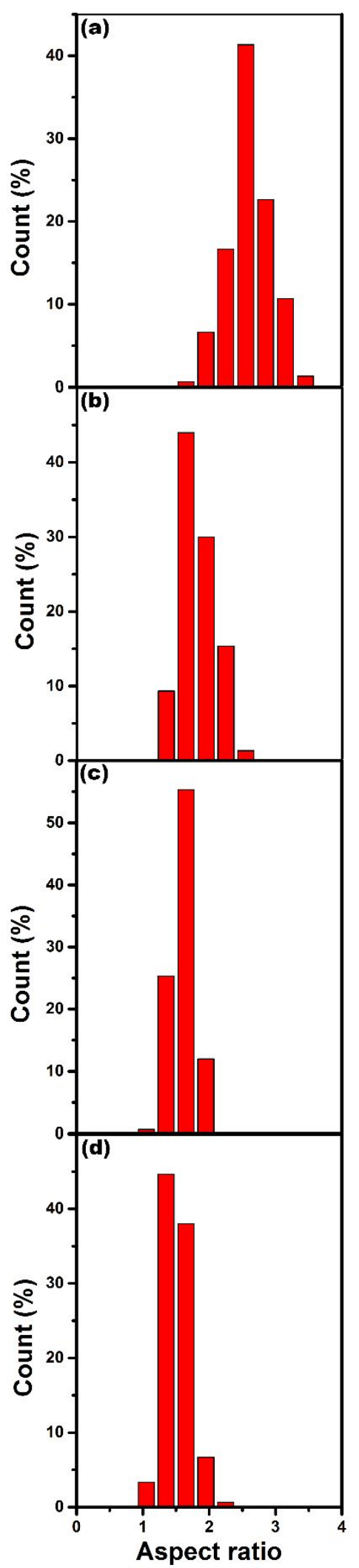

Figure S9. Histograms showing aspect ratio distributions for (a) ZB-ZnSe NRs, (b) ZB-ZnSe/ZnS core/shell NRs-1.0, (c) ZB-ZnSe/ZnS core/shell NRs-3.0, and (d) ZB-ZnSe/ZnS core/shell NRs-5.0. 


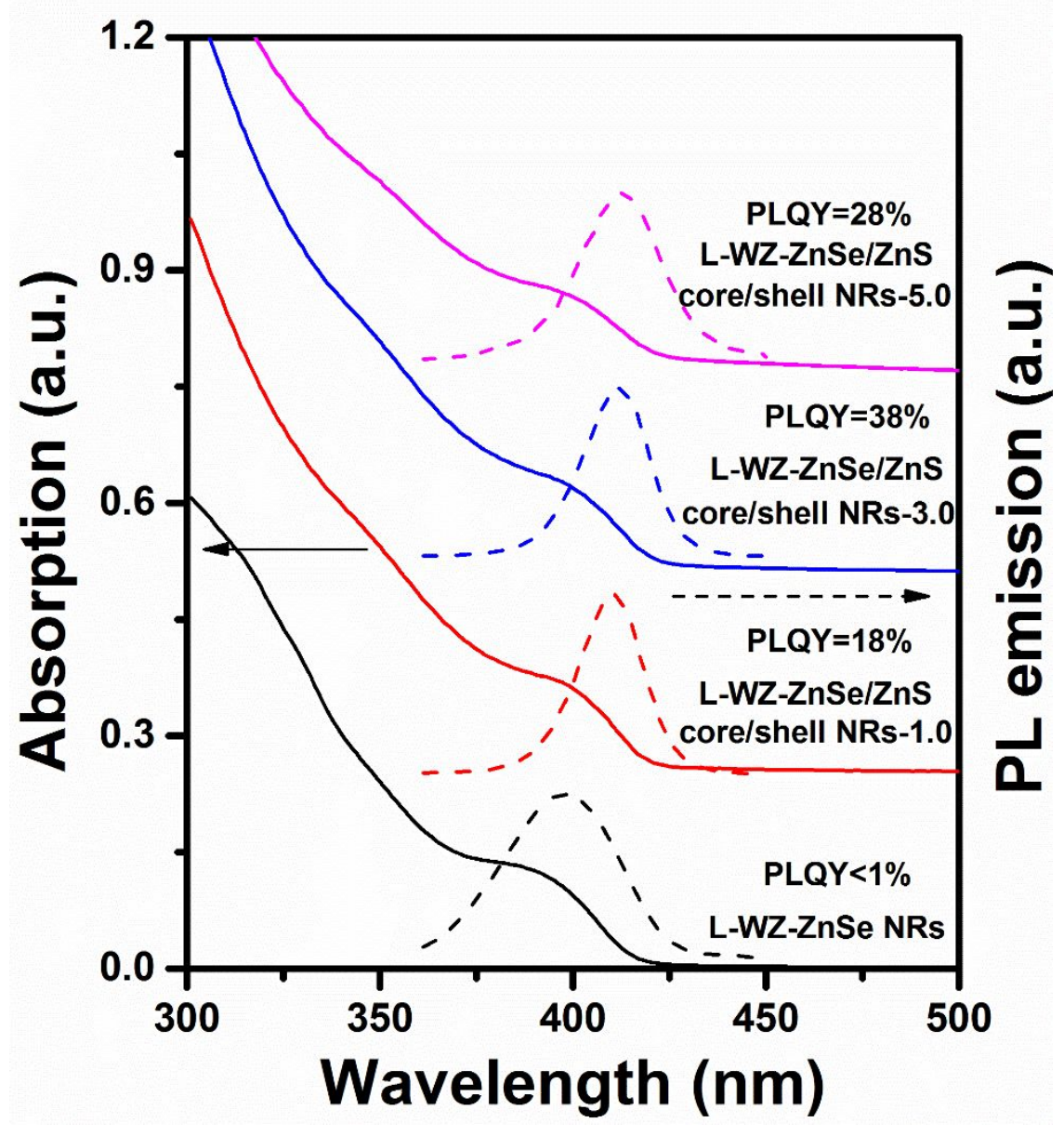

Figure S10. UV-vis absorption spectra (solid lines) and PL spectra (dashed lines) of the L-WZ-ZnSe NR cores and L-WZ-ZnSe/ZnS core/shell NRs; PLQYs for each sample is also provided. 


\section{Calculation of surface free energy of different facets in a cubic zinc-blende face- centered cubic (FCC) crystal structure ${ }^{1-3}$}

Considering the atoms in the surface regions of crystal structures, the surface free energy can be thought of as the energy to remove the atoms, relating to break the bonds of the atoms at the surface and thus to the energy of one bond for atoms. Then the energy of one bond for atoms in a crystal can be defined through the concept of sublimation energy.

For one mole of a crystal, there are $\mathrm{N}_{\mathrm{A}}$ atoms and at least $0.5 \mathrm{~N}_{\mathrm{A}}$ bonds will be formed amongst them. Taking the coordination number $(\mathrm{CN})$ into account, and assuming the number of nearest neighbor atoms as equal to $\mathrm{CN}$, there will be $\left(0.5 \mathrm{~N}_{\mathrm{A}} \mathrm{Z}\right.$ ( $\mathrm{Z}$ is the number of formula units in one unit cell, and $\mathrm{Z}=12$ in $\left.\mathrm{FCC}\right)$ ) bonds in one mole of a crystal.

The energy of one bond can be written as:

$$
e=\frac{\Delta H_{S}}{0.5 N_{A} Z},\left(\Delta H_{S} \text { is the molar enthalpy of sublimation }\right)
$$

\section{The (111) plane of the FCC lattice can be shown as:}

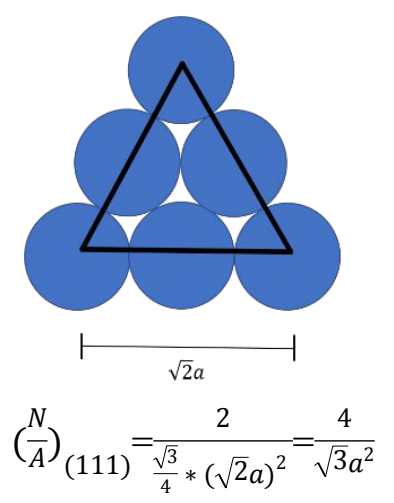

For the (111) plane of an FCC crystal, atoms at the surface have $\mathrm{CN}$ of 6 (nearest neighbor atoms), ${ }^{1}$ meaning that 3 bonds per atom should be broken at the (111) surface to remove one atom. Thus, the energy required for each atom to form a (111) surface in an FCC lattice can be given as:

$$
E_{(111) / \text { atom }}=(\text { energy of one bond }) \times(\text { number of bonds broken } / \text { atom })=3 \times \mathrm{e}=\frac{\Delta H_{S}}{2 N_{A}}
$$

The surface energy $E_{(111)}$ can be written as follows:

$E_{(111)}=($ Energy per surface atom $) \times($ number of surface atoms $/$ surface area $)=\frac{\Delta H_{S}}{2 N_{A}}\left(\frac{N}{A}\right)$

Thus, we obtain $E_{(111)}=\frac{\Delta H_{s}}{2 N_{A}}\left(\frac{4}{\sqrt{3} a^{2}}\right)=\frac{2 \Delta H_{s}}{\sqrt{3} N_{A} a^{2}}$ 


\section{The (110) plane in the FCC lattice can be shown as:}

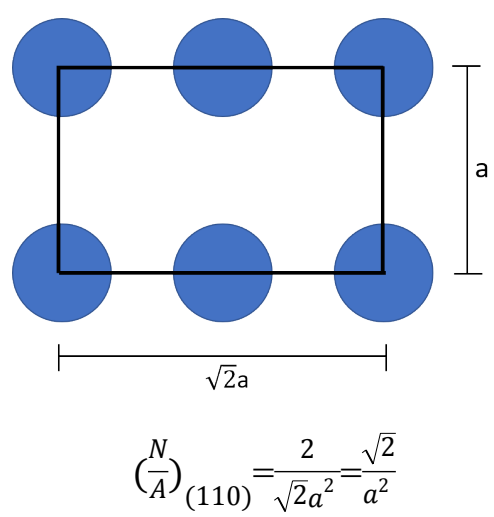

For the (110) plane of an FCC crystal, atoms at the surface have $\mathrm{CN}$ of 6 (nearest neighbor atoms), ${ }^{1}$ also meaning that 3 bonds per atom are broken at the surface of (110). Thus, the energy required for each atom to form a (110) surface in an FCC lattice can be given as:

$$
E_{(110) / \text { atom }}=(\text { energy of one bond }) \times(\text { number of bonds broken } / \text { atom })=3 \times e=\frac{\Delta H_{S}}{2 N_{A}}
$$

The surface energy $E_{(110)}$ can be written as follows:

$$
E_{(110)}=(\text { Energy per surface atom }) \times(\text { number of surface atoms } / \text { surface area })=\frac{\Delta H_{S}}{2 N_{A}}\left(\frac{N}{A}\right)
$$

Thus, we obtain $E_{(110)}=\frac{1 \Delta H_{s}}{2 N_{A}}\left(\frac{\sqrt{2}}{a^{2}}\right)=\frac{\sqrt{2} \Delta H_{S}}{N_{A} a^{2}}$

The ratio of surface energies for (111) and (110) planes in an FCC lattice is:

$$
\frac{E_{(111)}}{E_{(110)}}=\frac{2}{\sqrt{2}}=0.816
$$

The ratio of 0.816 means the surface energy of the (110) plane is higher than that of a (111) plane for an FCC lattice.

\section{References}

1. Wang, S. G.; Tian, E. K.; Lung, C. W. Surface Energy of Arbitrary Crystal Plane of BCC and FCC Metals.

J. Phys. Chem. Solids, 2000, 61, 1295-1300.

2. Chapter 6: Surface Energy. Edited by Prof. Yung-Jung Hsu (National Chiao Tung University).

3. Handout Supplement for the Ceramics Course. Edited by Prof. Yung-Jung Hsu (National Chiao Tung University). 